\title{
Correction to: Nest predation and the influence of habitat structure on nest predation of Wood Warbler Phylloscopus sibilatrix, a ground-nesting forest passerine
}

Paul E. Bellamy ${ }^{1}$ (1) Malcolm D. Burgess $^{1} \cdot$ John W. Mallord ${ }^{1} \cdot$ Andrew Cristinacce $^{1} \cdot$ Christopher J. Orsman $^{1}$. Tony Davis ${ }^{2}$. Philip V. Grice ${ }^{3}$. Elisabeth C. Charman ${ }^{1,4}$

Published online: 5 May 2018

(c) Dt. Ornithologen-Gesellschaft e.V. 2018

Correction to: Journal of Ornithology (2018) 159:493-506

https://doi.org/10.1007/s10336-017-1527-7

In the original publication of the article, there is a misalignment of the last two columns in Table 2. The correct Table 2 is provided below.

The original article can be found online at https://doi.org/10.1007/ s10336-017-1527-7.

Paul E. Bellamy paul.bellamy@rspb.org.uk

1 RSPB Centre for Conservation Science, The Lodge, Sandy, Bedfordshire SG19 2DL, UK

217 Orchards Way, West End, Southampton SO30 3FB, UK

3 Natural England, Mail Hub, County Hall, Spetchley Road, Worcester WR5 2NP, UK

4 Present Address: RSPB, 1 Sirius House, Amethyst Road, Newcastle Business Park, Newcastle-upon-Tyne NE4 7YL, UK 
Table 2 The identified predators from 95 predation events out of 144 Wood Warbler nests by study site monitored by nest camera

\begin{tabular}{|c|c|c|c|c|}
\hline & Dartmoor & New forest & Mid-Wales & Total \\
\hline \multicolumn{5}{|l|}{ Birds } \\
\hline $\begin{array}{l}\text { Jay } \\
\text { Garrulus glandarius }\end{array}$ & 7 & 5 & 18 & 30 \\
\hline $\begin{array}{l}\text { Buzzard } \\
\text { Buteo buteo }\end{array}$ & 8 & 1 & 4 & 13 \\
\hline $\begin{array}{l}\text { Sparrowhawk } \\
\text { Accipiter nisus }\end{array}$ & 4 & 1 & 2 & 7 \\
\hline $\begin{array}{l}\text { Tawny owl } \\
\text { Strix aluco }\end{array}$ & 0 & 2 & 0 & 2 \\
\hline $\begin{array}{l}\text { Great spotted woodpecker } \\
\text { Dendrocopus major }\end{array}$ & 0 & 0 & 1 (Plus one partial $\left.{ }^{\mathrm{a}}\right)$ & 1 \\
\hline \multicolumn{5}{|l|}{ Mammals } \\
\hline $\begin{array}{l}\text { Badger } \\
\text { Meles meles }\end{array}$ & 2 & 4 & 1 & 7 \\
\hline $\begin{array}{l}\text { Fox } \\
\text { Vulpes vulpes }\end{array}$ & 1 & 2 & 1 & 4 \\
\hline $\begin{array}{l}\text { Weasel } \\
\text { Mustela nivalis }\end{array}$ & 2 & 0 & 0 & 2 \\
\hline $\begin{array}{l}\text { Dog } \\
\text { Canis familiaris }\end{array}$ & 0 & 1 & 0 & 1 \\
\hline $\begin{array}{l}\text { Grey squirrel } \\
\text { Sciurus carolinensis }\end{array}$ & 1 & 2 & 0 & 3 \\
\hline Other rodents & 3 & 2 & 0 & 5 \\
\hline \multicolumn{5}{|l|}{ Other } \\
\hline $\begin{array}{l}\text { Adder } \\
\text { Vipera berus }\end{array}$ & 0 & 2 & 0 & 2 \\
\hline Unknown & 5 & 7 & 4 & 16 \\
\hline
\end{tabular}

${ }^{\text {a }}$ Partial predation is defined as a predator taking some nest contents with the remainder surviving and the nest attempt continuing 